\title{
Minimal rights based solidarity
}

\author{
Roland Iwan Luttens*
}

March, 2009

\begin{abstract}
In a model where individuals with different levels of skills exert different levels of effort, we propose to use individuals' minimal rights to divide an extra amount of income generated by a change in the skill profile. Priority is given to individuals with a positive minimal right which ensures that the way redistribution is performed depends on the total sum of income available in society. We characterize two families of Minimal Rights based Egalitarian mechanisms. One family guarantees each individual her claim when claims are feasible. The other family guarantees a non-negative income after redistribution for all individuals.
\end{abstract}

JEL Classification: D63.

Keywords: minimal rights, solidarity, compensation, claims, fairness.

\section{Introduction}

Suppose income inequalities are determined by unequal exerted effort levels and different innate skills. The goal of fair income redistribution is to guarantee an equal income for individuals exerting the same effort (the principle of compensation) and to perform equal income transfers to individuals with equal skills (the principle of natural reward). In an illuminating series of papers, Fleurbaey (1994, 1995a,b,c,d), Bossert (1995) and Bossert and Fleurbaey (1996) show that in many contexts there does not exist a redistribution mechanism that satisfies both the principle of compensation and the principle of natural reward simultaneously. Subsequently, by proposing different trade-offs between both principles,

\footnotetext{
${ }^{*}$ Postdoctoral Fellow of the Fund for Scientific Research - Flanders. SHERPPA (Ghent University) and CORE (Université catholique de Louvain). I am grateful to Dirk Van de gaer for stimulating discussions and to the Editor, two anonymous Referees, Alexander Cappelen, Geert Dhaene, Marc Fleurbaey, François Maniquet, Glenn Rayp, Erik Schokkaert, Bertil Tungodden and seminar/conference participants at LGS5 (Bilbao, 2007), Laboratoire d'Econométrie de l'Ecole Polytechnique (Paris, 2006), SSC\&W (Istanbul, 2006) and SED (Bodrum, 2006) for insightful comments. Additional financial support from the Interuniversity Attraction Poles Programme - Belgian State - Belgian Science Policy [Contract No. P6/07] is gratefully acknowledged. Address of correspondence: SHERPPA, Faculty of Economics and Business Administration, Ghent University, Tweekerkenstraat 2, 9000 Ghent, Belgium, tel: +32(0)92643487, fax: +32(0)92648996, roland.luttens@ugent.be.
} 
the literature has characterized many different families of fair redistribution mechanisms. Notably, most contributions have opted for a weakening of the principle of natural reward rather than for a weakening of the principle of compensation; see again Fleurbaey (1994, 1995a,b,c,d), Bossert (1995) and Bossert and Fleurbaey (1996) but also Iturbe (1997), Sprumont (1997) and Tungodden (2005). We refer to Fleurbaey and Maniquet (2007) for an extensive overview of the different families of fair redistribution mechanisms proposed. Two families play a benchmark role in this paper. It concerns (i) the family of Egalitarian Equivalent mechanisms owing to Pazner and Schmeidler (1978) and Fleurbaey (1995c) and (ii) the family of Proportionally Adjusted Equivalent mechanisms owing to Iturbe (1997). These two families are respectively characterized by Bossert and Fleurbaey (1996) and Iturbe (1997) in a very comprehensible way. First, the principle of compensation is strenghtened to the ethical principle of 'Solidarity' which can be traced back to the seminal contribution of Rawls (1971). Suppose that a change in the skill profile of society generates an extra amount (or a loss) of pre-tax income. Solidarity requires that all incomes change in the same direction when going from the final income distribution before the change in the skill profile to the final income distribution after the change in the skill profile. Second, Solidarity is strenghtened in 'Additive Solidarity' and 'Multiplicative Solidarity' successively. When going from the final income distribution before the change in the skill profile to the final income distribution after the change in the skill profile, Additive Solidarity requires that the absolute income differences remain constant whereas Multiplicative Solidarity requires that the relative income differences remain constant. Third, the principle of natural reward is weakened to the ethical principle of 'Equal Transfer for Reference Skill'. This axiom requires that equal income transfers are performed when the skill level of all individuals equals one particular 'reference' skill. The reference skill is assumed to be exogenously determined by the social planner. Then, a redistribution mechanism satisfies Additive Solidarity and Equal Transfer for Reference Skill if and only if it is an Egalitarian Equivalent mechanism and a redistribution mechanism satisfies Multiplicative Solidarity and Equal Transfer for Reference Skill if and only if it is a Proportionally Adjusted Equivalent mechanism.

In this paper, we propose a different strenghtening of the ethical principle of Solidarity. We propose to divide (take away) the income gain (loss), generated by a change in the skill profile, on the basis of the information contained in individuals' 'minimal rights'. The minimal right of an individual equals the amount that remains from the total sum of income when all other individuals have received their 'claim'. The claim of an individual depends on her exerted effort level but does not depend on her innate skill. The minimal right of an individual is not allowed to be negative nor to exceed the individual's claim. In the division of the income gain (loss), priority is given to an individual with a higher claim, which is due to higher exerted effort, once the total sum of income exceeds the individual-specific threshold where her minimal right becomes positive. The crucial distinction between the ethical principle of 'Minimal Rights 
based Solidarity' proposed in this paper and the ethical principles of Additive or Multiplicative Solidarity is that the way solidarity is performed under Minimal Rights based Solidarity depends on the particular economy considered, i.e. depends on the total sum of income available. Loosely speaking, the way we divide (take away) the income gain (loss) in an economy with a high total sum of income is considerably different from the way we divide (take away) the income gain (loss) in an economy with a low total sum of income.

Given the introduction of claims, our analysis shows similarities with the literature on bankruptcy problems and surplus sharing problems. In a bankruptcy problem, a fixed amount of money must be allocated on the basis of monetary claims that sum up to more than can be divided. The objective is to design allocation mechanisms that associate with each claims problem a division of the amount available over the claimants. We refer to Thomson (2003) for an extensive survey of the literature on conflicting claims problems. In a surplus sharing problem, an amount of money that exceeds the total sum of claims must be divided over all claimants. An extensive survey on surplus sharing problems is Moulin (2002). It is not hard to see that the fair income redistribution problem can formally be interpreted as a conflicting claims (surplus sharing) problem where the total sum of income before redistribution has to be divided over a population of claimants. Readers familiar with the conflicting claims/surplus sharing literature will note that the Egalitarian Equivalent mechanism is formally similar to (an unconstrained version of) the Equal Losses/Equal surplus sharing mechanism (see Herrero et al. (1999)) and that the Proportionally Adjusted Equivalent mechanism is formally similar to the Proportional mechanism, probably the most popular mechanism in practice for both conflicting claims and surplus sharing problems. Nevertheless, many of the ethical principles imposed in the conflicting claims literature and the fair income redistribution literature are very different. We stress that this paper aims at contributing to the literature on fair income distribution. A deeper exploration of the intersection between both strands of the literature is postponed for future research.

The paper is organized as follows. In the next section, we first present the model (section 2.1). Then, we state different ethical principles, introduce the families of Egalitarian Equivalent mechanisms and Proportionally Adjusted Equivalent mechanisms formally and discuss their characterizations (section 2.2). In section 3, we first argue why we believe that there is room for a new principle of solidarity. We introduce the notion of minimal rights and propose to use minimal rights to divide an extra amount of income generated by a change in the skill profile (section 3.1). Then, we state two solidarity axioms that, when imposed together, constitute the central Minimal Rights based Solidarity principle of this paper (section 3.2). Subsequently, we characterize two families of Minimal Rights based Egalitarian mechanisms that implement this solidarity principle (section 3.3). A concluding discussion (section 4) is provided. 


\section{Preliminaries}

\section{$2.1 \quad$ Model}

The fair monetary compensation model used in this paper is a one-dimensional version of the quasi-linear model in Fleurbaey and Maniquet (2007) which is due to Bossert (1995). Denote $N=\{1, \ldots, n\}$ the finite population of size $n \geq 2$. Let $x \in \mathbb{R}$ be an amount of transferable resource. The characteristic which elicits compensation, hereafter called 'skill', is $y \in Y$ and $Y$ is an interval of $\mathbb{R}$. Denote $y_{N}=\left(y_{1}, \ldots, y_{n}\right)$ the skill profile in the population. The characteristic which does not elicit compensation, hereafter called 'effort', is $z \in Z$ and $Z$ is an interval of $\mathbb{R}$. Effort is not influenced by redistribution as incentive issues are not taken up in the model. Denote $z_{N}=\left(z_{1}, \ldots, z_{n}\right)$ the effort profile in the population. Without loss of generality, we assume that individuals are ranked such that $z_{1} \geq \ldots \geq z_{n}$. An economy $e=\left(y_{N}, z_{N}\right)$ is the pair of characteristics' profiles. Denote $\mathcal{E}$ the set of economies.

We assume that utility functions are quasi-linear, i.e. $u\left(x_{i}, y_{i}, z_{i}\right)=x_{i}+v\left(y_{i}, z_{i}\right)$. In the context of this paper $u: \mathbb{R} \times Y \times Z \rightarrow \mathbb{R}$ measures a monetary outcome, namely final income after redistribution. The function $v: Y \times Z \rightarrow \mathbb{R}_{++}$ describes the pre-tax income function. We assume that $v$ is continuous and strictly increasing in $y$ and $z$. Furthermore, we assume that $v$ is not additively separable in $y$ and $z$, i.e. $v\left(y_{i}, z_{i}\right)$ cannot be written as $v_{1}\left(y_{i}\right)+v_{2}\left(z_{i}\right) .{ }^{1}$ Denote $R=\sum_{i \in N} v\left(y_{i}, z_{i}\right)$ the total sum of pre-tax incomes.

Let the transferable resource $x_{i}$ be an element of an allocation $x_{N}=\left(x_{1}, \ldots, x_{n}\right)$ $\in \mathbb{R}^{n}$. We assume that the total amount to be distributed is 0 , such that we are looking at a redistribution problem. An allocation for the economy $e \in \mathcal{E}$ is feasible when $\sum_{i \in N} x_{i}=0$. The set of feasible allocations for an economy $e \in \mathcal{E}$ is denoted $F(e)$. Notice that all feasible allocations are Pareto efficient since we ruled out free disposal in the definition of feasibility. An allocation mechanism is denoted $S: \mathcal{E} \rightarrow \mathbb{R}^{n}$, such that, for all $e \in \mathcal{E}, S(e) \subseteq F(e)$. Let $\mathcal{S}$ be the set of all allocation mechanisms.

Let $\tilde{y} \in Y$ be the reference skill. We assume throughout the paper that this constant parameter is exogenously determined by the social planner. Denote $v\left(\tilde{y}, z_{i}\right)$ the reference income of individual $i$. It equals the pre-tax income that an individual would receive when exerting her effort level $z_{i}$ but having skill $\tilde{y}$ instead of her own skill $y_{i}$. Denote $\tilde{R}=\sum_{i \in N} v\left(\tilde{y}, z_{i}\right)$ the total sum of reference incomes.

We introduce a function $g: Z \rightarrow \mathbb{R}_{++}$that assigns to each individual a claim that depends on the individual's effort but does not depend on the individual's skill. Hence, two individuals with identical effort but different skills (and hence

\footnotetext{
${ }^{1}$ When $v$ is additively separable in $y$ and $z$, a natural way to redistribute income (that satisfies both the principal of compensation and the principal of natural reward) is to make each individual's income after redistribution equal to the average contribution of $y_{N}$ plus the individual contribution of $z_{i}$ in the income generating process (Bossert (1995)).
} 
different pre-tax incomes) have identical claims in the redistribution problem. We assume that $g$ is continuous and strictly increasing in $z$. Denote $\mathfrak{g}$ the claims vector and $C=\sum_{i \in N} g\left(z_{i}\right)$ the total sum of claims. A conflicting claims problem is a pair $(\mathfrak{g}, R) \in \mathbb{R}_{++}^{n} \times \mathbb{R}_{++}$, such that $C \geq R$. A surplus sharing problem is a pair $(\mathfrak{g}, R) \in \mathbb{R}_{++}^{n} \times \mathbb{R}_{++}$, such that $C<R$. One particular example to model claims within the context of fair income redistribution is to use reference incomes as claims, i.e. $g\left(z_{i}\right)=v\left(\tilde{y}, z_{i}\right)$ for all $i \in N$ (see infra).

\subsection{Axioms and allocation mechanisms}

Throughout the paper, we consider different axioms that can generically be described as solidarity axioms. In general, solidarity axioms consider the effect of a change in one individual's skill on the allocation. Consider two skill profiles $y_{N}=\left(y_{1}, \ldots, y_{k}, \ldots, y_{n}\right)$ and $y_{N}^{\prime}=\left(y_{1}^{\prime}, \ldots, y_{k}^{\prime}, \ldots, y_{n}^{\prime}\right)$, where, for all $j$ in $N \backslash\{k\}, y_{j}$ equals $y_{j}^{\prime}$. Let $e^{\prime}=\left(y_{N}^{\prime}, z_{N}\right)$ and $R^{\prime}=\sum_{i \in N} v\left(y_{i}^{\prime}, z_{i}\right)$. Denote the change in total pre-tax income $\Delta_{R}=R^{\prime}-R$. Without loss of generality, we assume throughout the paper that $e^{\prime}$ yields more pre-tax income than $e$ and hence $\Delta_{R} \in \mathbb{R}_{++}$. Solidarity axioms state how these extra resources should be divided over the population. The weakest form of solidarity, which could be traced back to Rawls (1971), requires that a change in the skill profile (weakly) affects all agents' incomes after redistribution in the same direction. We consider two stronger versions of solidarity. The first axiom requires that all incomes change equally. An allocation mechanism $S$ satisfies 'Additive Solidarity' $(A S$, Bossert (1995)) if:

for all $e, e^{\prime} \in \mathcal{E}$, for all $x_{N} \in S(e)$ and all $x_{N}^{\prime} \in S\left(e^{\prime}\right)$, $x_{i}^{\prime}+v\left(y_{i}^{\prime}, z_{i}\right)-\left(x_{i}+v\left(y_{i}, z_{i}\right)\right)=x_{j}^{\prime}+v\left(y_{j}^{\prime}, z_{j}\right)-\left(x_{j}+v\left(y_{j}, z_{j}\right)\right)$ for all $i, j \in N$.

The second axiom requires that all individuals' outcomes change proportionally. An allocation mechanism $S$ satisfies 'Multiplicative Solidarity' ( $M S$, Iturbe (1997)) if:

for all $e, e^{\prime} \in \mathcal{E}$, for all $x_{N} \in S(e)$ and all $x_{N}^{\prime} \in S\left(e^{\prime}\right)$, $\left(x_{i}^{\prime}+v\left(y_{i}^{\prime}, z_{i}\right)\right)\left(x_{j}+v\left(y_{j}, z_{j}\right)\right)=\left(x_{i}+v\left(y_{i}, z_{i}\right)\right)\left(x_{j}^{\prime}+v\left(y_{j}^{\prime}, z_{j}\right)\right)$ for all $i, j \in N$.

We refer to Fleurbaey and Maniquet (2007) for a proof that $A S$ and $M S$ imply the principle of compensation (see introduction) under the assumption that the allocation mechanism is anonymous, i.e. transfers depend on relevant characteristics and not on individuals' names.

The following axiom is a weak version of the principle of natural reward (see introduction) since it only applies the principle of natural reward to economies where all skills are equal to the reference skill. In these economies no redistribution is performed. An allocation mechanism $S$ satisfies 'Equal Transfer for Reference Skill' (ETRS, Bossert and Fleurbaey (1996)) if:

for all $e \in \mathcal{E}$ and for all $x_{N} \in S(e)$, if $y_{i}=\tilde{y}$ for all $i \in N$, then $x_{i}=0$ for all $i \in N$. 
We also introduce an axiom that requires that all individuals receive their claim when claims are feasible. An allocation mechanism $S$ satisfies 'Claims Feasibility' if:

for all $e \in \mathcal{E}$ and for all $x_{N} \in S(e)$, if $R=C$, then $x_{i}=-v\left(y_{i}, z_{i}\right)+g\left(z_{i}\right)$ for all $i \in N$.

It is easy to see that Claims Feasibility implies ETRS when reference incomes are used as claims (i.e. when $g\left(z_{i}\right)=v\left(\tilde{y}, z_{i}\right)$ for all $\left.i \in N\right)$.

We define two families of allocation mechanisms. It concerns a) the family of Egalitarian Equivalent mechanisms due to Pazner and Schmeidler (1978) and Fleurbaey (1995) and b) the family of Proportionally Adjusted Equivalent mechanisms due to Iturbe (1997). Within each family, a particular mechanism is described by the particular choice of reference skill.

a) The $\tilde{y}$-Egalitarian Equivalent mechanism $\left(S_{\tilde{y} E E}\right)$ allocates resources as follows:

for all $e \in \mathcal{E}$ and for all $i \in N,\left(x_{i}\right)_{S_{\tilde{y} E E}}=-v\left(y_{i}, z_{i}\right)+v\left(\tilde{y}, z_{i}\right)+\frac{1}{n}(R-\tilde{R})$.

b) The $\tilde{y}$-Proportionally Adjusted Equivalent mechanism $\left(S_{\tilde{y} P A E}\right)$ allocates resources as follows:

for all $e \in \mathcal{E}$ and for all $i \in N,\left(x_{i}\right)_{S_{\tilde{y} P A E}}=-v\left(y_{i}, z_{i}\right)+\frac{R}{\tilde{R}} v\left(\tilde{y}, z_{i}\right)$.

These two families are characterized in the literature as follows:

1) An allocation mechanism $S$ satisfies ETRS and $A S$ if and only if it is an $S_{\tilde{y} E E}($ Bossert and Fleurbaey (1996)).

2) An allocation mechanism $S$ satisfies $E T R S$ and $M S$ if and only if it is an $S_{\tilde{y} P A E}$ (Iturbe (1997)).

Figure 1 illustrates for a given $\tilde{y}$ and for every value of $R$ the income distributions under $S_{\tilde{y} E E}$ and $S_{\tilde{y} P A E}$ for an economy with four individuals whose reference incomes are in a ratio of $6: 4: 2: 1$. A glance at the income distributions provides a clear interpretation of the axioms used in the characterization results. As both redistribution mechanisms satisfy the axiom of ETRS, income is redistributed such that every individual receives her reference income when $R$ equals $\tilde{R}$. As a consequence of Additive Solidarity, the absolute income inequality remains constant under $S_{\tilde{y} E E}$ when $R$ changes (full line). As a consequence of Multiplicative Solidarity, the relative income inequality remains constant under $S_{\tilde{y} P A E}$ when $R$ changes (dotted line). In comparing two income distributions with the same mean, we say that one income distribution is more equal than another income distribution when the former is obtained from the latter by performing a series of (Pigou-Dalton) rich-to-poor transfers that do not entail rank reversals. From figure 1, we see that income distributions under $S_{\tilde{y} P A E}$ are more equal than income distributions under $S_{\tilde{y} E E}$ when $R<\tilde{R}$, while the reverse holds when $R>\tilde{R}$. 


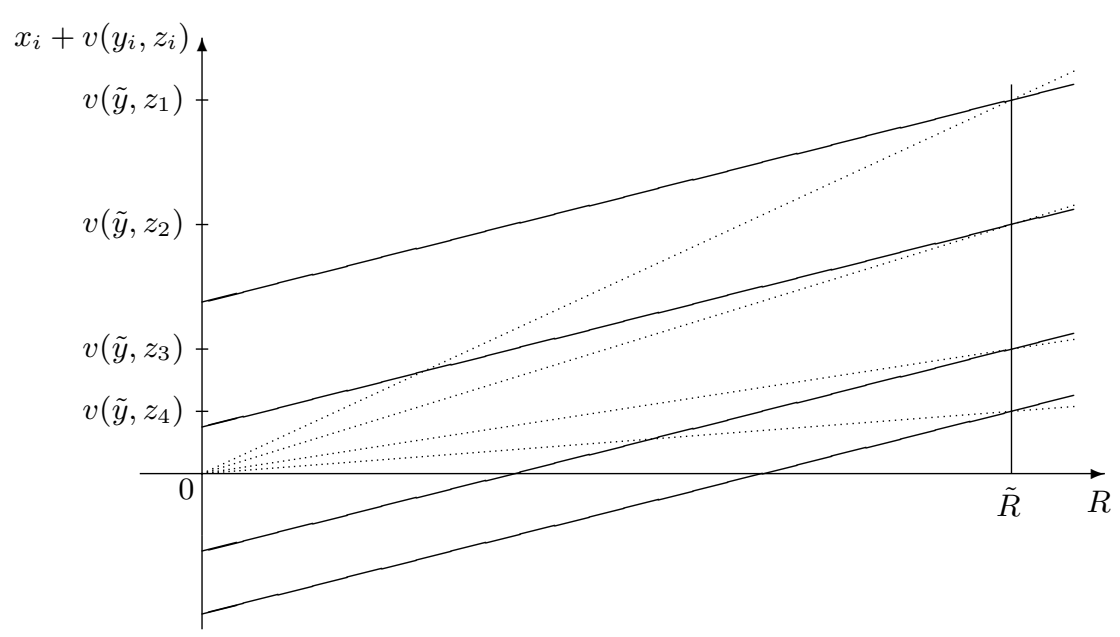

Figure 1: Income distributions under $S_{\tilde{y} E E}$ and $S_{\tilde{y} P A E}$

\section{Minimal rights based solidarity}

The previous section shows how closely the choice of a particular family of allocation mechanisms depends on the choice which solidarity principle to implement. In this section, we first critically reconsider Additive Solidarity and Multiplicative Solidarity and argue why there is room for a different solidarity principle (section 3.1). We introduce the concept of minimal rights and propose to use the information of individuals' minimal rights to divide the extra amount of income generated by a change in the skill profile. We then propose new solidarity axioms that express this Minimal Rights based Solidarity principle (section 3.2) and discuss two different families of allocation mechanisms and their respective income distributions that implement this Minimal Rights based Solidarity principle (section 3.3).

\subsection{Solidarity depending on the total sum of income.}

The central idea behind solidarity is that an individual who experienced a change in her skill should not alone bear the consequences of this change. However, the ethical principles of Additive and Multiplicative Solidarity are much stronger. Suppose that due to a change in the skill profile an extra amount of resources $\Delta_{R}$ needs to be divided. Additive Solidarity requires that the extra resources are divided equally over all individuals. A number of objections can be raised against this particular realization of solidarity:

1) By dividing the extra resources equally, any mechanism that satisfies $A S$ (like $\left.S_{\tilde{y} E E}\right)$ can be criticized for not taking differences in exerted effort into account. 
2) Any mechanism that satisfies $A S$ requires that solidarity is borne by all individuals. This is, however, not a necessary requirement to implement solidarity through redistribution. For example, it could be desirable to implement a redistribution mechanisms that is able to 'protect' the poorest individuals from the burdens of solidarity.

3) Any mechanism that satisfies $A S$ implements this solidarity principle independent of the total sum of income. This is, also, not a necessary requirement to implement solidarity through redistribution. For example, consider the same economy as in figure 1. Suppose that a social planner wants to design a redistribution mechanism that satisfies ETRS. On top of that, she has a concern for the income inequalities that arise after redistribution (she wants the post-tax income distribution as equal as possible). Suppose that she only considers $A S$ and $M S$ as solidarity principles. From figure 1, we conclude that her preferred solidarity principle is $M S$ when $R<\tilde{R}$, while it is $A S$ when $R>\tilde{R}$.

Objections 2 and 3 can also be raised against Multiplicative Solidarity. Concerning objection $1, M S$ does take differences in effort into account but it does so in one particular way. More precisely, any mechanism that satisfies $M S$ (like $S_{\tilde{y} P A E}$ ) divides the extra resources proportionally to individuals' reference incomes. $^{2}$

In what follows, we introduce a new principle of solidarity. Differences in effort are taken into account when dividing the extra resources, but need not necessarily be expressed through differences in reference incomes. More generally, differences in effort are reflected through differences in claims. Moreover, our precise realization of solidarity does not only depend on the claims vector but also on the total sum of pre-tax income available. In other words, we take, besides $\mathfrak{g}$, also $R$ into account when dividing the extra resources. It will turn out that, for some amounts of $R$, solidarity will not be borne by all individuals. Let us make our solidarity principle more precise.

We propose to base the division of extra resources on individuals' minimal rights, a concept originating from seminal contributions of O'Neill (1982) and Aumann and Maschler (1985) in the conflicting claims literature. ${ }^{3}$

Definition (minimal rights) The minimal right of an individual equals the amount that remains from the total sum of pre-tax income when all other individuals have received their claim. However, the minimal right is not allowed to be negative nor to exceed the individual's own claim.

Formally, the minimal right of an individual $i$ equals:

$$
m_{i}(\mathfrak{g}, R)=\min \left(g\left(z_{i}\right), \max \left(R-C_{-i}, 0\right)\right)
$$

\footnotetext{
${ }^{2}$ I.e., $\frac{x_{i}^{\prime}+v\left(y_{i}^{\prime}, z_{i}\right)-\left(x_{i}+v\left(y_{i}, z_{i}\right)\right)}{x_{j}^{\prime}+v\left(y_{j}^{\prime}, z_{j}\right)-\left(x_{j}+v\left(y_{j}, z_{j}\right)\right)}=\frac{v\left(\tilde{y}, z_{i}\right)}{v\left(\tilde{y}, z_{j}\right)}$ for all $i, j \in N$.

${ }^{3}$ Minimal rights should not be confused with the concept of equal rights introduced in Maniquet (1998). In a model with production, an allocation mechanism guarantees an equal right when every individual weakly prefers her bundle over her best choice from a common opportunity set.
} 
where

$$
C_{-i}=\sum_{j \in N \backslash\{i\}} g\left(z_{j}\right) \text { for all } i \in N
$$

Figure 2 illustrates the concept of minimal rights. As long as $R$ is smaller than $C_{-1}$, all minimal rights are zero. When $R$ exceeds $C_{-1}$, the minimal right of the individual with effort level $z_{1}$ becomes strictly positive. When $R$ exceeds $C_{-2}$, the minimal right of the individual with effort level $z_{1}$ exceeds $C_{-2}-C_{-1}$ and the minimal right of the individual with effort level $z_{2}$ becomes strictly positive. As $R$ increases, more and more individuals start to get a strictly positive minimal right and when $R$ exceeds $C_{-n}$ all minimal rights are strictly positive. When $R$ equals $C$, every individual has a minimal right equal to her claim. When $R$ exceeds $C$, minimal rights do not change anymore, so in all surplus sharing problems $m_{i}(\mathfrak{g}, R)$ equals $g\left(z_{i}\right)$ for all $i$ in $N$.

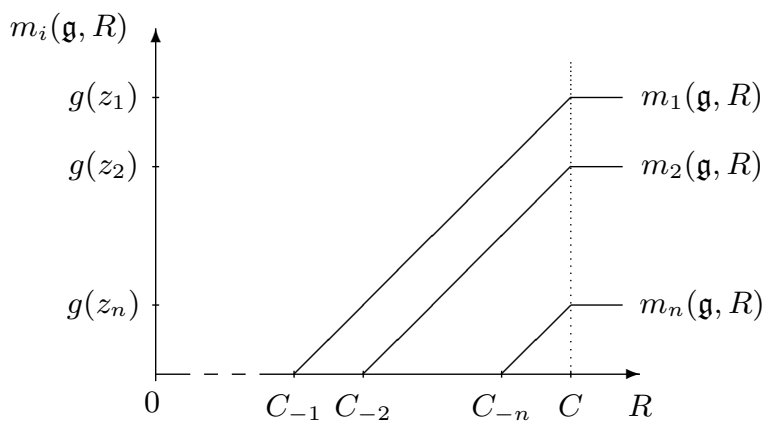

Figure 2: Minimal rights

An individual with a higher claim (due to higher effort) has a higher minimal right, provided that the latter is different from zero. A strictly positive minimal right could be given the interpretation of an uncontested part of the total sum of income, an amount of resources that the population as a whole concedes to a particular individual on the basis of their claims. Suppose that $R$ equals $C_{-2}$. Then all individuals agree that, once they have received their claim, the individual with effort level $z_{1}$ deserves at least her minimal right, i.e. $C_{-2}-C_{-1}$, of $R$. However, minimal rights of different individuals might overlap. Suppose that $R$ equals $C_{-3}$. Although there is agreement that the amount $C_{-2}-C_{-1}$ of $R$ should only go to the individual with effort level $z_{1}$, the amount $C_{-3}-C_{-2}$ of $R$ is conceded to both the individual with effort level $z_{1}$ and the individual with effort level $z_{2}$.

We envision a solidarity principle based on minimal rights as follows. When total income changes due to a change in the skill profile, none, some or all minimal rights may change, depending on $\mathfrak{g}, R$ and $\Delta_{R}$. Denote $\Delta m_{i}\left(\mathfrak{g}, R, \Delta_{R}\right)=$ $m_{i}\left(\mathfrak{g}, R+\Delta_{R}\right)-m_{i}(\mathfrak{g}, R)$ the change in the minimal right of individual $i$ due 
to a change of total pre-tax income equal to $\Delta_{R}$. We propose to base the division of the extra resources on the changes in minimal rights. Consider the following examples. Suppose that $R$ is smaller than $C_{-1}$. Take any income distribution as given and assume that the skill profile improves. First, suppose that $R^{\prime}$ is still smaller than $C_{-1}$. All minimal rights remain zero: $R^{\prime}$ is completely contested and there is no agreement that any individual deserves a particular amount of the extra resources. In this case, we propose to divide the extra resources equally over all individuals. Second, suppose that $R^{\prime}$ equals an amount between $C_{-1}$ and $C_{-2}$. The minimal right of the individual with effort level $z_{1}$ equals $R^{\prime}-C_{-1}$, while the minimal rights of all other individuals remain zero. Now $R^{\prime}$ is not completely contested: there is agreement that the individual with effort level $z_{1}$ deserves $R^{\prime}-C_{-1}$. In this case, we propose to give the uncontested amount $R^{\prime}-C_{-1}$ to the individual with effort level $z_{1}$. The rest, $C_{-1}-R$, is divided equally over all individuals. Third, suppose that $R^{\prime}$ equals an amount between $C_{-2}$ and $C_{-3}$. The income level $C_{-1}$ is completely contested. As before, we propose to divide the amount $C_{-1}-R$ equally over all individuals. Next, the amount $C_{-2}-C_{-1}$ is conceded to the individual with effort level $z_{1}$ and we propose that the latter receives this amount entirely. Finally, the amount of $R^{\prime}-C_{-2}$ is conceded to both the individual with effort level $z_{1}$ and the individual with effort level $z_{2}$. We propose to divide this amount equally between them. The iterative reasoning of the third example can be applied for higher values of $R^{\prime}$, at least until $R^{\prime}$ equals $C_{-n}$. When $R^{\prime}$ exceeds $C_{-n}$, the amount $R^{\prime}-C_{-n}$ is conceded to all individuals. Hence, we propose to divide $R^{\prime}-C_{-n}$ again equally over all individuals. The following section presents two axioms that, when imposed together, embody the above sketched idea of solidarity based on minimal rights.

\subsection{Solidarity axioms}

The first axiom weakens the axiom of Additive Solidarity by requiring an equal treatment of two individuals in the allocation of the extra resources only when their minimal rights change equally. A redistribution mechanism $S$ satisfies 'Additive Solidarity for Equal Changes in Minimal Rights' $\left(A S^{*}\right)$ if:

for all $e, e^{\prime} \in \mathcal{E}$, for all $x_{N} \in S(e)$ and all $x_{N}^{\prime} \in S\left(e^{\prime}\right)$, and for all $i, j \in N$,

$$
\begin{aligned}
& \text { if } \Delta m_{i}\left(\mathfrak{g}, R, \Delta_{R}\right)=\Delta m_{j}\left(\mathfrak{g}, R, \Delta_{R}\right), \\
& \text { then } x_{i}^{\prime}+v\left(y_{i}^{\prime}, z_{i}\right)-\left(x_{i}+v\left(y_{i}, z_{i}\right)\right)=x_{j}^{\prime}+v\left(y_{j}^{\prime}, z_{j}\right)-\left(x_{j}+v\left(y_{j}, z_{j}\right)\right) .
\end{aligned}
$$

The second axiom gives priority in the allocation of the extra resources to individuals with a strictly positive change in their minimal rights over individuals whose minimal rights remain constant. The axiom presented here is very weak. It only focuses on the case where the population can be partitioned in two disjoint subsets: the subset of individuals whose change in minimal rights equals the change in total pre-tax income and the subset of individuals whose minimal rights do not change at all. The axiom states that, when the former subset is non-empty, the extra resources should be divided only among individuals 
belonging to that former subset. By excluding individuals in performing solidarity, the axiom is clearly incompatible with $A S$ or $M S$. Formally, denote $N_{1}=\left\{i \in N \mid \Delta m_{i}\left(\mathfrak{g}, R, \Delta_{R}\right)=\Delta_{R}\right\}$ the subset of individuals whose change in minimal rights equals the change in total pre-tax income. Denote $N_{1}^{c}$ the complement of $N_{1}$ (with respect to $N$ ). Denote $N_{2}=\left\{i \in N \mid \Delta m_{i}\left(\mathfrak{g}, R, \Delta_{R}\right)=0\right\}$ the subset of individuals whose minimal rights do not change. A redistribution mechanism $S$ satisfies 'Priority' if:

for all $e, e^{\prime} \in \mathcal{E}$, for all $x_{N} \in S(e)$ and all $x_{N}^{\prime} \in S\left(e^{\prime}\right)$,

if $\Delta_{R}$ is such that $N_{1} \neq \varnothing$ and $N_{1}^{c}=N_{2}$,

then $\sum_{i \in N_{1}}\left(x_{i}^{\prime}+v\left(y_{i}^{\prime}, z_{i}\right)-x_{i}-v\left(y_{i}, z_{i}\right)\right)=\Delta_{R}$.

Both axioms together imply the paper's central solidarity idea of Minimal Rights based Solidarity. ${ }^{4}$ In other words, by imposing $A S^{*}$ and Priority, the division of any amount of extra resources over the population is implied. The intuition is the following. As $v$ is continuous and strictly increasing in $y$, any $\Delta_{R}$ can be divided in specific subchanges that are constructed in such a way that, for each of them, the population $N$ is partitioned in $N_{1}$ and $N_{2}$. If, for a subchange, $N_{1}=N$ or $N_{2}=N, A S^{*}$ guarantees an equal division of the subchange over all individuals. If, for a subchange, $N_{1}$ and $N_{2}$ are both non-empty, Priority guarantees that the subchange is divided only among the individuals in $N_{1}$ and $A S^{*}$ guarantees that the subchange is divided equally over the latter individuals. The complete division of $\Delta_{R}$ is obtained by adding up the divisions of the subchanges. We state Minimal Rights based Solidarity formally in Appendix.

\subsection{Minimal Rights based Egalitarian mechanisms}

Fair income redistribution mechanisms that satisfy Minimal Rights based Solidarity are denoted Minimal Rights based Egalitarian mechanisms. In order to characterize one particular family of Minimal Rights based Egalitarian mechanisms, it suffices to combine Minimal Rights based Solidarity independently with one other axiom that for one specific $R$ implies one specific income distribution. An obvious candidate is the axiom of Claims Feasibility. Combining

\footnotetext{
${ }^{4}$ One referee suggested to consider a weakening of Multiplicative Solidarity. A redistribution mechanism $S$ satisfies 'Multiplicative Solidarity for Equal Changes in Minimal Rights' $\left(M S^{*}\right)$ if:

for all $e, e^{\prime} \in \mathcal{E}$, for all $x_{N} \in S(e)$ and all $x_{N}^{\prime} \in S\left(e^{\prime}\right)$, and for all $i, j \in N$,$$
\text { if } \Delta m_{i}\left(\mathfrak{g}, R, \Delta_{R}\right)=\Delta m_{j}\left(\mathfrak{g}, R, \Delta_{R}\right) \text {, }
$$$$
\text { then }\left(x_{i}^{\prime}+v\left(y_{i}^{\prime}, z_{i}\right)\right)\left(x_{j}+v\left(y_{j}, z_{j}\right)\right)=\left(x_{i}+v\left(y_{i}, z_{i}\right)\right)\left(x_{j}^{\prime}+v\left(y_{j}^{\prime}, z_{j}\right)\right) \text {. }
$$

Combining $M S^{*}$ with Priority leads to a multiplicative version of Minimal Rights based Solidarity. In our opinion, $M S^{*}$ has slightly less appeal than $A S^{*}$. We find it more arbitrary to proportionally (rather than equally) reward equal changes in minimal rights, especially as the latter exemplify equal concessions of total income. We do not explore this multiplicative version of Minimal Rights based Solidarity througout the paper but we comment on it in the concluding discussion (see footnote 5).
} 
Minimal Rights based Solidarity and Claims Feasibility characterizes the following mechanism.

A Minimal Rights based Egalitarian mechanism $S_{g M R E / C F}$ allocates resources as follows:

for all $e \in \mathcal{E}$,

(1) when $C_{-n} \leq R$ :

$\left(x_{i}\right)_{S_{g M R E / C F}}=-v\left(y_{i}, z_{i}\right)+g\left(z_{i}\right)+\frac{R-C}{n}$ for all $i$ in $N$,

(2) when, for $k \leq n-1, C_{-k} \leq R<C_{-(k+1)}$ :

$\left(x_{i}\right)_{S_{g M R E / C F}}=-v\left(y_{i}, z_{i}\right)+g\left(z_{i}\right)+\frac{C_{-n}-C}{n}-\sum_{h=k+1}^{n-1}\left(\frac{C_{-(h+1)}-C_{-h}}{h}\right)+\frac{R-C_{-(k+1)}}{k}$

for all $i \in\{1, \ldots, k\}$ and

$\left(x_{j}\right)_{S_{g M R E / C F}}=-v\left(y_{j}, z_{j}\right)+g\left(z_{j}\right)+\frac{C_{-n}-C}{n}-\sum_{h=j}^{n-1}\left(\frac{C_{-(h+1)}-C_{-h}}{h}\right)$

for all $j \in\{k+1, \ldots, n-1\}$ and

$\left(x_{n}\right)_{S_{g M R E / C F}}=-v\left(y_{n}, z_{n}\right)+g\left(z_{n}\right)+\frac{C_{-n}-C}{n}$,

(3) when $R<C_{-1}$ :

$\left(x_{i}\right)_{S_{g M R E / C F}}=-v\left(y_{i}, z_{i}\right)+g\left(z_{i}\right)+\frac{C_{-n}-C}{n}-\sum_{h=i}^{n-1}\left(\frac{C_{-(h+1)}-C_{-h}}{h}\right)+\frac{R-C_{-1}}{n}$

for all $i \in\{1, \ldots, n-1\}$ and

$\left(x_{n}\right)_{S_{g M R E / C F}}=-v\left(y_{n}, z_{n}\right)+g\left(z_{n}\right)+\frac{C_{-n}-C}{n}+\frac{R-C_{-1}}{n}$.

Proposition $1 S=S_{g M R E / C F} \Leftrightarrow S$ satisfies Minimal Rights based Solidarity and Claims Feasibility.

The proof of proposition 1 can be found in Appendix.

Figure 3 depicts for every value of $R$ the income distributions under an $S_{g M R E / C F}$ for an economy with four individuals whose claims are in a ratio of 6:4:2:1.

Because $S_{g M R E / C F}$ satisfies Claims Feasibility, income is redistributed such that every individual receives her claim when $R$ equals $C$. When, due to a change in the skill profile, $R$ becomes higher than $C$, every individual receives her claim plus an equal part of $R-C$. When $R$ becomes lower than $C$, every individual receives an income that is lower than her claim. As long as $R$ is higher than $C_{-4}$, the loss of total pre-tax income is equally borne by all individuals. Similarly, when $R$ becomes smaller than $C_{-1}$, the loss $C_{-1}-R$ is borne equally by all individuals. But, for any $R$ between $C_{-3}$ and $C_{-4}$, the income of the poorest 


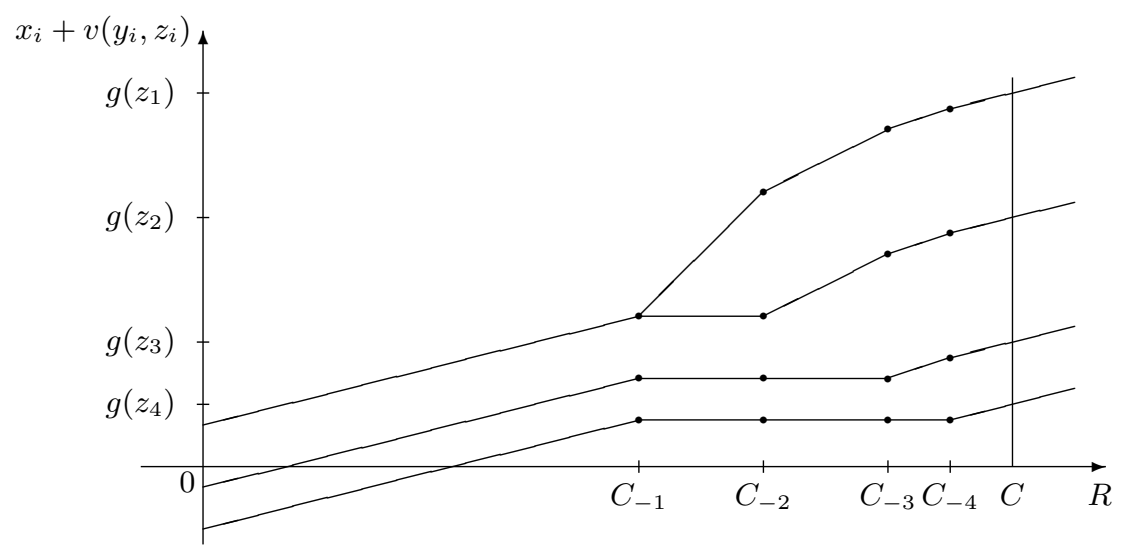

Figure 3: Income distributions under $S_{g M R E / C F}$

individual remains constant. Similarly, for any $R$ between $C_{-2}$ and $C_{-3}$, the incomes of the poorest and second poorest individual remain constant and, for any $R$ between $C_{-1}$ and $C_{-2}$, the richest individual alone is saddled with the entire cost of keeping the incomes of all other individuals constant. These observations demonstrate that, for some values of $R$, Minimal Rights based Solidarity implies that solidarity is not always borne by all individuals.

In general, $S_{g M R E / C F}$ does not satisfy ETRS. For example, when $\tilde{R}<C_{-1}$, the post-tax incomes of the individuals with effort levels $z_{1}$ and $z_{2}$ are equal under $S_{g M R E / C F}$ and cannot correspond with the unequal reference incomes $v\left(\tilde{y}, z_{1}\right)$ and $v\left(\tilde{y}, z_{2}\right)$. However, when reference incomes are used as claims $(\tilde{R}=C)$, Claims Feasibility can be replaced by the weaker axiom ETRS in the characterization of $S_{g M R E / C F}$.

Corollary 1 Suppose $g\left(z_{i}\right)=v\left(\tilde{y}, z_{i}\right)$ for all $i \in N$. Then $S=S_{g M R E / C F} \Leftrightarrow S$ satisfies Minimal Rights based Solidarity and ETRS.

A debatable property of $S_{g M R E / C F}$ (and of $S_{\tilde{y} E E}$ as well), illustrated in Figure 3 , is that the poorest individuals might end up with a negative income after redistribution when $R$ is sufficiently low. Our ethical intuition may lead us to consider a minimal amount of redistribution that we at least want to perform. Suppose that the poorest in society could not satisfy their basic needs when they receive a negative income after redistribution. Society wants to exclude this possibility in every situation by incorporating the requirement of a non-negative income after redistribution for all individuals in the construction of the redistribution mechanism. A redistribution mechanism $S$ satisfies 'Participation' (Maniquet (1998)) if:

for all $e \in \mathcal{E}$, for all $x_{N} \in S(e), x_{i}+v\left(y_{i}, z_{i}\right) \geq 0$ for all $i \in N$. 
An implication of Participation is that, when $R$ converges to zero, all incomes should also converge to zero. Combining Participation with Minimal Rights based Solidarity characterizes the following mechanism.

A Minimal Rights based Egalitarian mechanism $S_{g M R E / P}$ allocates resources as follows:

for all $e \in \mathcal{E}$,

(1) when $R<C_{-1}$ :

$\left(x_{i}\right)_{S_{g M R E / P}}=-v\left(y_{i}, z_{i}\right)+\frac{R}{n}$ for all $i$ in $N$,

(2) when, for $k \leq n-1, C_{-k} \leq R<C_{-(k+1)}$ :

$\left(x_{i}\right)_{S_{g M R E / P}}=-v\left(y_{i}, z_{i}\right)+\frac{C_{-1}}{n}+\sum_{h=i}^{k}\left(\frac{C_{-(h+1)}-C_{-h}}{h}\right)+\frac{R-C_{-(k+1)}}{k}$

for all $i \in\{1, \ldots, k\}$ and

$\left(x_{j}\right)_{S_{g M R E / P}}=-v\left(y_{j}, z_{j}\right)+\frac{C_{-1}}{n}$ for all $j \in\{k+1, \ldots, n\}$,

(3) when $C_{-n} \leq R$ :

$\left(x_{i}\right)_{S_{g M R E / P}}=-v\left(y_{i}, z_{i}\right)+\frac{C_{-1}}{n}+\sum_{h=i}^{n-1}\left(\frac{C_{-(h+1)}-C_{-h}}{h}\right)+\frac{R-C_{-n}}{n}$

for all $i \in\{1, \ldots, n-1\}$ and

$\left(x_{n}\right)_{S_{g M R E / P}}=-v\left(y_{n}, z_{n}\right)+\frac{C_{-1}}{n}+\frac{R-C_{-n}}{n}$.

Proposition $2 S=S_{g M R E / P} \Leftrightarrow S$ satisfies Minimal Rights based Solidarity and Participation.

The proof of proposition 2 can be found in Appendix.

Figure 4 illustrates, for the same economy as in figure 3, the income distributions under $S_{g M R E / P}$.

An equal distribution of income prevails as long as all minimal rights are zero. More generally, when $R$ is lower than or equal to $C_{-i}$ for some $i$ in $N$, all individuals with $z$ lower than or equal to $z_{i}$ receive the same income. Clearly, as $S_{g M R E / C F}$ and $S_{g M R E / P}$ are different mechanisms, Participation is incompatible with Claims Feasibility when Minimal Rights based Solidarity is imposed. Furthermore, when reference incomes are used as claims, Participation is also incompatible with ETRS when Minimal Rights based Solidarity is imposed. 


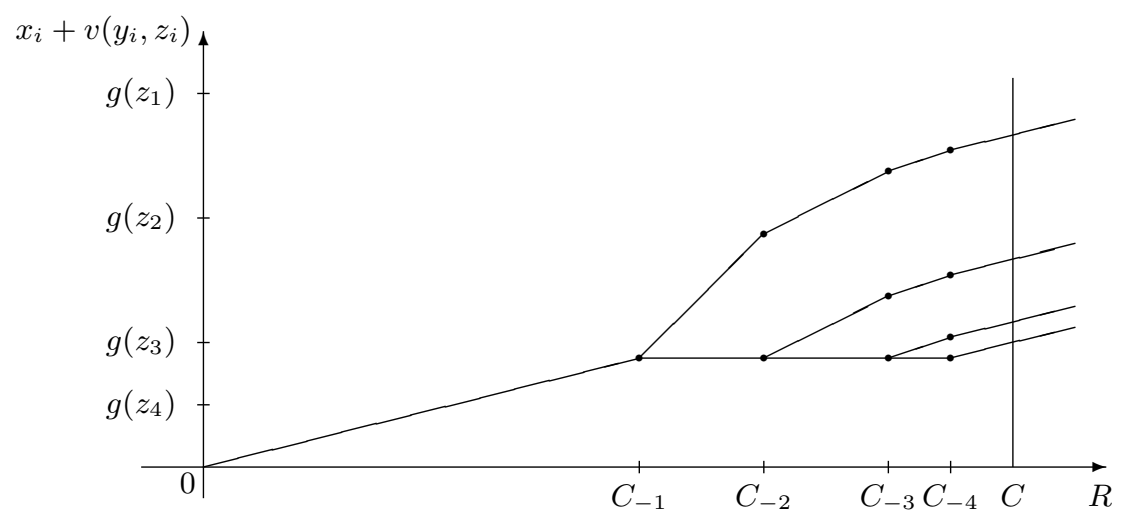

Figure 4: Income distributions under $S_{g M R E / P}$

\section{Concluding discussion}

In this paper, we propose to use individuals' minimal rights to divide an extra amount of income generated by a change in the skill profile in a model where individuals with different levels of skills exert different levels of effort. Priority is given to individuals with a positive minimal right, which ensures that the way solidarity is performed depends on the total sum of income available in society. We characterize two families of Minimal Rights based Egalitarian mechanisms. $S_{g M R E / C F}$ guarantees each individual her claim when claims are feasible. $S_{g M R E / P}$ guarantees a non-negative income after redistribution for all individuals.

We end the paper by discussing (i) the post-tax income inequalities that arise under the different mechanisms and (ii) the incompatibility between Participation and Claims Feasibility when Minimal Rights based Solidarity is imposed. The tightness of propositions 1 and 2 is demonstrated in Appendix.

(i) An $S_{g M R E / P}$ redistributes incomes very equally. It is easy to check that, for every value of $R$, the income distribution under $S_{g M R E / P}$ is more equal than the income distribution under $S_{g M R E / C F}$.

When reference incomes are used as claims, some additional conclusions can be drawn from comparing figures 1 and 3 :

- In economies where every individual has a strictly positive minimal right $\left(C_{-n}<R\right)$, an $S_{g M R E / C F}$ redistributes income just like an $S_{\tilde{y} E E}$. Both mechanisms redistribute income more equally than an $S_{\tilde{y} P A E}$ in all surplus sharing problems. 
- In economies where some but not all minimal rights are strictly positive $\left(C_{-1}<\right.$ $R \leq C_{-n}$ ), the income distribution under an $S_{g M R E / C F}$ is more equal than the income distribution under an $S_{\tilde{y} E E}$.

- In economies where all minimal rights are zero $\left(R \leq C_{-1}\right)$, absolute income inequalities remain constant under an $S_{g M R E / C F}$ and under an $S_{\tilde{y} E E}$ when $R$ changes, but incomes are more equally distributed under the former mechanism.

(ii) The incompatibility between Participation and Claims Feasibility under Minimal Rights based Solidarity (imposing $A S^{*}$ and Priority together), is due to $A S^{*}$ rather than Priority. ${ }^{5}$

When Priority is dropped, imposing Participation, $A S^{*}$ and Claims Feasibility still leads to an incompatibility. This is best explained as follows. Start from an income distribution $(0,0, \ldots, 0)$ when $R$ converges to zero (Participation). Now suppose $R^{\prime}$ equals $C$. Then, by Claims Feasibility, individual $n$ should receive $g\left(z_{n}\right)$. Note that the subchanges of $\Delta_{R}$ for which all minimal rights change equally are $C_{-1}\left(=\sum_{i=2}^{n} g\left(z_{i}\right)\right)$ and $C-C_{-n}\left(=g\left(z_{n}\right)\right)$. Hence, by $A S^{*}$, individual $n$ is certain to receive $\frac{1}{n}\left(\sum_{i=2}^{n} g\left(z_{i}\right)+g\left(z_{n}\right)\right)$. Denote $A$ the amount that individual $n$ further receives from the division of $C_{-n}-C_{-1}$. Then, for any $A \in\left[0, C_{-n}-C_{-1}\right]$ and given our assumption that claims are increasing in $z$, the condition $\frac{1}{n}\left(\sum_{i=2}^{n} g\left(z_{i}\right)+g\left(z_{n}\right)\right)+A=g\left(z_{n}\right)$ cannot hold in general.

When $A S^{*}$ is dropped, Participation, Priority and Claims Feasibility are compatible but do not characterize a unique family of redistribution mechanisms. The intuition is clear: Participation describes a precise income distribution when $R$ converges to zero, Claims Feasibility describes a precise income distribution when $R$ equals $C$ and Priority only describes precise income transfers between $C_{-1}$ and $C_{-2}$ (only the income of individual with effort $z_{1}$ changes), which together is not enough to describe a precise allocation mechanism for all values of $R$. For all values of $R \leq C$, we illustrate in figure 5 for the same economy as in figures 3 and 4 (i) the most equal income distribution (upper panel) and (ii) the most unequal income distribution (lower panel) that can be

\footnotetext{
${ }^{5}$ This conclusion cannot be drawn if we consider the multiplicative version of Minimal Rights based Solidarity (imposing $M S^{*}$ and Priority together) and use reference incomes as claims. When Priority is dropped, Participation, ETRS and $M S^{*}$ are compatible (because $S_{\tilde{y} P A E}$ is the unique mechanism satisfying Participation, ETRS and $M S$ and $M S^{*}$ is weaker than $M S$ ). However, imposing Participation, ETRS, $M S^{*}$ and Priority leads to an incompatibility. This is best explained as follows. Denote $S_{\text {prop-g } M R E / P}$ the mechanism that satisfies Participation, $M S^{*}$ and Priority. Start from an income distribution $(0,0, \ldots, 0)$ (Participation). By $M S^{*}, S_{\tilde{y} P A E}=S_{\text {prop-g } M R E / P}$ for any $R$ between 0 and $C_{-1}$ and, furthermore, relative income inequality remains constant for any $R$ between $C_{-n}$ and $C$. But, by the interplay of $M S^{*}$ and Priority, for any $R$ between $C_{-1}$ and $C_{-n}$, the income distribution under $S_{\text {prop-gMRE/P }}$ is obtained from the income distribution under $S_{\tilde{y} P A E}$ by a series of poorto-rich transfers (i.e. by transfers from individuals with a zero change in minimal rights to individuals with a strictly positive change in minimal rights). As $S_{\tilde{u} P A E}$ redistributes income more equally than $S_{\text {prop-g } M R E / P}$, the latter does not satisfy $E T R S$.
} 
obtained from a redistribution mechanism that satisfies Participation, Priority and Claims Feasibility.
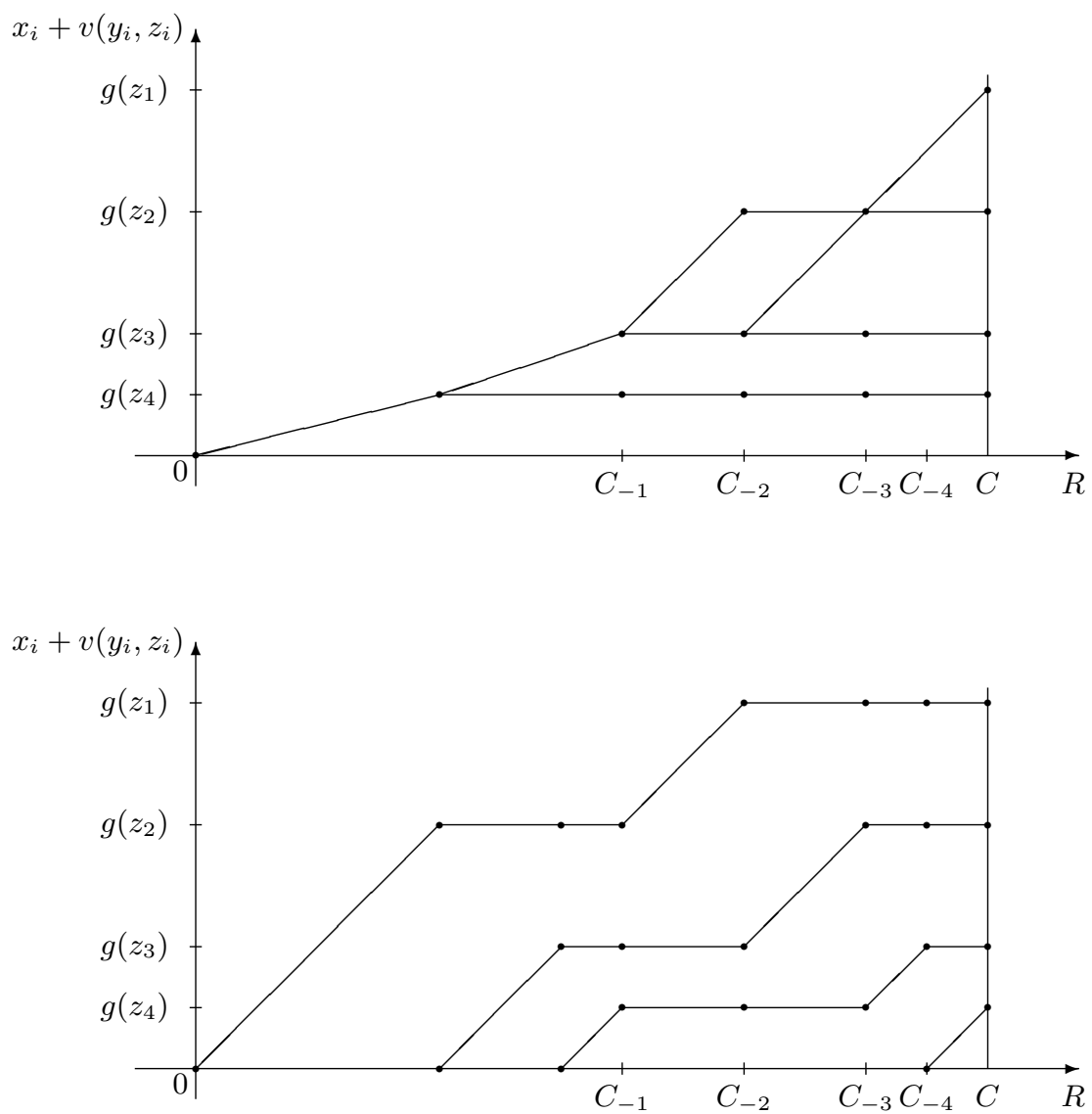

Figure 5: The most equal and the most unequal income distributions under a mechanism satisfying Participation, Priority and Claims Feasibility

\section{Appendix: Proofs}

First, Lemma 1 states formally the solidarity idea of Minimal Rights based Solidarity, i.e. how a change in the total sum of pre-tax income is divided over the population such that the axioms of $A S^{*}$ and Priority are satisfied. Second, propositions 1 and 2 are proved. Third, the tightness of propositions 1 and 2 is demonstrated. 
Lemma 1 (Minimal Rights based Solidarity): Consider $\Delta_{R}>0$ due to a skill change from $y_{i}$ to $y_{i}^{\prime}$ of an individual $i$ in $N$. Denote $d_{i}=x_{i}^{\prime}+v\left(y_{i}^{\prime}, z_{i}\right)-$ $\left(x_{i}+v\left(y_{i}, z_{i}\right)\right) ; \sum_{i \in N} d_{i}=\Delta_{R}$. One of five possible situations occurs:

(1) when $R^{\prime} \leq C_{-1}$ or $C_{-n} \leq R$ :

$d_{i}=\frac{\Delta_{R}}{n}$ for all $i$ in $N$,

(2) when, for $k \leq n-1, C_{-k} \leq R<R^{\prime}<C_{-(k+1)}$ :

$d_{i}=\frac{\Delta_{R}}{k}$ for all $i \in\{1, \ldots, k\}$ and

$d_{j}=0$ for all $j \in\{k+1, \ldots, n\}$,

(3) when, for $k \leq n-1, C_{-k} \leq R<C_{-(k+1)}$ and, for $2 \leq l \leq n, C_{-l} \leq R^{\prime}<$ $C_{-(l+1)}$ and $k<l: 6$

$d_{i}=\frac{C_{-(k+1)}-R}{k}+\sum_{h=k+1}^{l-1}\left(\frac{C_{-(h+1)}-C_{-h}}{h}\right)+\frac{R^{\prime}-C_{-l}}{l}$ for all $i \in\{1, \ldots, k\}$ and

$d_{j}=\sum_{h=j}^{l-1}\left(\frac{C_{-(h+1)}-C_{-h}}{h}\right)+\frac{R^{\prime}-C_{-l}}{l}$ for all $j \in\{k+1, \ldots, l-1\}$ and

$d_{l}=\frac{R^{\prime}-C_{-l}}{l}$ and

$d_{q}=0$ for all $q \in\{l+1, \ldots, n\}$,

(4) when $R \leq C_{-1}$ and, for $l \leq n-1, C_{-l} \leq R^{\prime}<C_{-(l+1)}$ :

$d_{i}=\frac{C_{-1}-R}{n}+\sum_{h=i}^{l}\left(\frac{C_{-(h+1)}-C_{-h}}{h}\right)+\frac{R^{\prime}-C_{-(l+1)}}{l}$ for all $i \in\{1, \ldots, l\}$ and

$d_{j}=\frac{C_{-1}-R}{n}$ for all $j \in\{l+1, \ldots, n\}$,

(5) when $R \leq C_{-1}$ and $C_{-n} \leq R^{\prime}$ :

$d_{i}=\frac{C_{-1}-R}{n}+\sum_{h=i}^{n-1}\left(\frac{C_{-(h+1)}-C_{-h}}{h}\right)+\frac{R^{\prime}-C_{-n}}{n}$ for all $i \in\{1, \ldots, n-1\}$ and

$d_{n}=\frac{C_{-1}-R}{n}+\frac{R^{\prime}-C_{-n}}{n}$.

Proof. Suppose the antecedent of (1) is true. None of the minimal rights change and the division of $\Delta_{R}$ is obtained by $A S^{*}$. Suppose the antecedent of (2) is true. There are two groups of individuals. For individuals 1 to $k$ minimal rights change equally. For individuals $k+1$ to $n$ minimal rights do not change. $A S^{*}$ implies that, within each group, all individuals are treated in the same way. Priority requires that the first group receives $\Delta_{R}$. The division of $\Delta_{R}$ then follows straightforwardly. Suppose the antecedent of (3) is true. As $v$ is continuous and strictly increasing in $y$, there exist for individual $i$ unique

${ }^{6}$ When $l=n$, define $C_{-(l+1)}=+\infty$. 
intermediary skill levels $\hat{y}_{i}^{k}, \hat{y}_{i}^{k+1}, \ldots, \hat{y}_{i}^{l}$ such that, ceteris paribus, the total sum of pre-tax income equals $C_{-k}, C_{-(k+1)}, \ldots, C_{-l}$ respectively. Now consider skill changes from $y_{i}$ to $\hat{y}_{i}^{k}, \hat{y}_{i}^{k}$ to $\hat{y}_{i}^{k+1}, \ldots, \hat{y}_{i}^{l}$ to $y_{i}^{\prime}$ such that the total change of pretax income equals $C_{-k}-R, C_{-(k+1)}-C_{-k}, \ldots, R^{\prime}-C_{-l}$ respectively. For each of these subchanges there are two groups of individuals: $(i)$ a group whose change in minimal rights is equal to the subchange and (ii) a group whose minimal rights do not change. $A S^{*}$ implies that within each group all individuals are treated in the same way, whereas Priority requires that the first group receives the subchange. Hence, the subchange is equally divided among the individuals of the first group. The division of $\Delta_{R}$ is obtained from applying $A S^{*}$ and Priority to the division of these subchanges. The division of $\Delta_{R}$ under (4) and (5) is obtained by similar reasoning as in (3).

\section{Proof of proposition 1}

$S=S_{g M R E / C F} \Leftrightarrow S$ satisfies $A S^{*}$, Priority and Claims Feasibility.

Proof. We only prove $(\Leftarrow)$. Consider an economy $\bar{e}=\left(\bar{y}_{N}, z_{N}\right)$ for which $\bar{R}=C$. By Claims Feasibility, $\bar{x}_{i}=-v\left(y_{i}, z_{i}\right)+g\left(z_{i}\right)$ for all $i$ in $N$ and individuals' final incomes are equal to their claims. Call this the 'initial income distribution'. Rather than successively considering (at most) $n$ changes from $\bar{y}_{N}$ to $y_{N}$ and using Lemma 1 successively to divide the intermediate subchanges in total pre-tax income (a process where in many cases previous subchanges in total pre-tax income would cancel out), we immediately use Lemma 1 to divide $\Delta_{R}$. The transfers of (1) in the definition of $S_{g M R E / C F}$ then follow from adding to the initial income distribution the transfers described in case (1) in lemma 1 . The transfers of (2) then follow from subtracting of the initial income distribution the transfers described in case (3) with $l=n$ in lemma 1 . The transfers of (3) then follow from subtracting of the initial income distribution the transfers described in case (5) in lemma 1.

\section{Proof of proposition 2}

$S=S_{g M R E / P} \Leftrightarrow S$ satisfies $A S^{*}$, Priority and Participation.

Proof. We only prove $(\Leftarrow)$. Participation requires that, when $R$ converges to zero, all incomes also converge to zero. Let $(0,0, \ldots, 0)$ be the initial income distribution. Now, use Lemma 1 to divide $\Delta_{R}$. The transfers of (1) in the definition of $S_{g M R E / P}$ are then described in case (1) in lemma 1. The transfers of (2) are then described in case (4) in lemma 1. The transfers of (3) are then described in case (5) in lemma 1.

\section{Tightness results}

The axioms used in the characterization of $S_{g M R E / C F}$ are independent. For example, when reference incomes are used as claims, $S_{\tilde{y} E E}$ satisfies Claims Feasibility and $A S^{*}$ but violates Priority. All mechanisms that implement one of the two income distributions depicted in figure 5 for an economy with four individuals whose claims are in a ratio of 6:4:2:1 satisfy Claims Feasibility and Priority but violate $A S^{*} . S_{g M R E / P}$ satisfies $A S^{*}$ and Priority but violates 
Claims Feasibility. The axioms used in the characterization of $S_{g M R E / P}$ are also independent. A straightforward example of a mechanism that satisfies Participation and $A S^{*}$ but violates Priority is the equal division of income. All mechanisms that implement one of the two income distributions depicted in figure 5 for an economy with four individuals whose claims are in a ratio of 6:4:2:1

satisfy Participation and Priority but violate $A S^{*}$. Finally, $S_{g M R E / C F}$ satisfies $A S^{*}$ and Priority but violates Participation.

\section{References}

[1] Aumann, R. and Maschler, M. (1985), Game theoretic analysis of a bankruptcy problem from the Talmud, Journal of Economic Theory 36, 195-213.

[2] Bossert, W. (1995), Redistribution mechanisms based on individual factors, Mathematical Social Sciences 29, 1-17.

[3] Bossert, W. and Fleurbaey, M. (1996), Redistribution and compensation, Social Choice and Welfare 13, 343-355.

[4] Fleurbaey, M. (1994), On fair compensation, Theory and Decision 36, 277307.

[5] Fleurbaey, M. (1995a), Equality and responsibility, European Economic Review 39, 683-689.

[6] Fleurbaey, M. (1995b), Three solutions for the compensation problem, Journal of Economic Theory 65, 505-521.

[7] Fleurbaey, M. (1995c), The requisites of equal opportunity, in W.A. Barnett, H. Moulin, M. Salles and N. Schofield (eds), Social Choice, Welfare and Ethics, Cambridge University Press.

[8] Fleurbaey, M. (1995d), Equal opportunity or equal social outcome?, Economics and Philosophy 11, 25-55.

[9] Fleurbaey, M. and Maniquet, F. (2007), Compensation and responsibility, forthcoming in K.J. Arrow, A.K. Sen and K. Suzumura (eds.), Handbook of Social Choice and Welfare, Volume 2, North-Holland: Elsevier.

[10] Herrero, C., Maschler, M. and Villar, A. (1999), Individual rights and collective responsibility: the rights-egalitarian solution, Mathematical Social Sciences 37, 59-77.

[11] Iturbe-Ormaetxe, I. (1997), Redistribution and individual characteristics, Review of Economic Design 3, 45-55.

[12] Maniquet, F. (1998) An equal right solution to the compensationresponsibility dilemma, Mathematical Social Sciences 35(2), 185-202. 
[13] Moulin, H. (2002), Axiomatic cost and surplus sharing, in K.J. Arrow, A.K. Sen and K. Suzumura (eds.), Handbook of Social Choice and Welfare, Volume 1, North-Holland: Elsevier.

[14] O'Neill, B. (1982), A problem of rights arbitration from the Talmud, Mathematical Social Sciences 2, 345-371.

[15] Pazner, E. and Schmeidler, D. (1978), Egalitarian equivalent allocations: A new concept of economic equity, Quarterly Journal of Economics 92, 671-687.

[16] Rawls, J. (1971), A Theory of Justice, Cambridge: Harvard University Press.

[17] Sprumont, Y. (1997), Balanced egalitarian redistribution of income, Mathematical Social Sciences 33, 185-201.

[18] Thomson, W. (2003), Axiomatic and game-theoretic analysis of bankruptcy and taxation problems: a survey, Mathematical Social Sciences 45, 249-297.

[19] Tungodden, B. (2005), Responsibility and redistribution: The case of first best taxation, Social Choice and Welfare 24, 33-44. 